\title{
A NEW QUANTITY IN RIEMANN-FINSLER GEOMETRY*
}

\author{
XIAOHUAN MO \\ Key Laboratory of Pure and Applied Mathematics \\ School of Mathematical Sciences \\ Peking University, Beijing 100871, China \\ e-mail:moxh@pku.edu.cn \\ ZHONGMIN SHEN \\ Department of Mathematical Sciences \\ Indiana University-Purdue University Indianapolis \\ 402 N. Blackford Street \\ Indianapolis, IN 46202-3216, USA \\ e-mail: zshen@math.iupui.edu \\ and HUAIFU LIU \\ College of Applied Science \\ Beijing University of Technology, Beijing 100022, China \\ e-mail:liufu369@emails.bjut.edu.cn
}

(Received 13 August 2011; accepted 19 January 2012; first published online 30 March 2012)

\begin{abstract}
In this note, we study a new Finslerian quantity $\hat{C}$ defined by the Riemannian curvature. We prove that the new Finslerian quantity is a non-Riemannian quantity for a Finsler manifold with dimension $n=3$. Then we study Finsler metrics of scalar curvature. We find that the $\hat{C}$-curvature is closely related to the flag curvature and the $H$-curvature. We show that $\hat{C}$-curvature gives, a measure of the failure of a Finsler metric to be of weakly isotropic flag curvature. We also give a simple proof of the Najafi-Shen-Tayebi' theorem.
\end{abstract}

1991 Mathematics Subject Classification. 58E20.

1. Introduction. In Finsler geometry, there are several important geometric quantities: the flag curvature, the (mean) Cartan torsion and the (mean) Berwald curvature, etc. (cf. $[\mathbf{6}, \mathbf{1 0}]$ ). In [1], H. Arbar-Zadeh considered a Finslerian quantity $H$, which is obtained from the mean Berwald curvature by the covariant horizontal differentiation along geodesics. Arbar-Zadeh proved that for a Finsler metric of scalar flag curvature with dimension $\geq 3$, the flag curvature is constant on the manifold if and only if $H=0$.

Recently, a great progress has been made in studying Finsler metrics of weakly isotropic flag curvature. These Finsler metrics are of scalar curvature whose flag curvature is in a special form $K=\theta / F+\sigma$ where $\theta$ is a 1 -form and $\sigma$ is a scalar function on $M$. Finsler metrics of weakly isotropic flag curvature not only include Finsler metrics of constant flag curvature, but also include Finsler metrics of (almost) isotropic $S$-curvature and of scalar flag curvature $[4,6,13]$. Cheng and Shen have

*The first author is supported by the NNSFC (11071005) and the second author is supported in part by NSF (DMS-0810159). 
classified Finsler metrics of Randers type with weakly isotropic flag curvature via the navigation problem in Riemannian manifolds $[\mathbf{5}, \mathbf{1 2}]$. Chen-Zhao constructed explicitly Finsler metrics are of scalar curvature such that they are not of weakly isotropic flag curvature [3]. A natural task for us is to give a geometric quantity on a Finsler manifold, which characterises the Finsler metrics of weakly isotropic flag curvature.

In this paper, we find the desired quantity (see Section 2 below) and call it the $\hat{C}$-curvature. We show that the $\hat{C}$-curvature gives a measure of the failure of an $n$ dimensional Finsler metric of scalar curvature to be of weakly isotropic flag curvature if $n \geq 3$. Precisely we prove the following:

TheOREM 1.1. Let $(M, F)$ be an $n(\geq 3)$-dimensional Finsler manifold of scalar curvature with flag curvature $K(x, y)$. Then $K$ is weakly isotropic if and only if the $\hat{C}$-curvature vanishes.

Recall that a Finsler metric $F$ is said to be of scalar curvature if the flag curvature $K=K(x, y)$ is a scalar function on the slit tangent bundle $T M \backslash\{0\}$. For these Finsler metrics, we establish an equation between the flag curvature $K$, the $\hat{C}$-curvature $\hat{C}$ and the $H$-curvature $H$ (see Proposition 3.2 below), therefore the $\hat{C}$-curvature is subtly related to the flag curvature and the $H$-curvature.

Recently, Najafi-Shen-Tayebi extended Arbar-Zadeh's characterisation for Finsler metrics of constant flag curvature and proved the following $[\mathbf{8}, \mathbf{1 1}, \mathbf{1 3}]$ :

THEOREM 1.2. Let $F$ be a Finsler metric of scalar flag curvature on an $n(\geq 3)$ dimensional manifold $M$. For a 1-form $\theta$, the flag curvature is weakly isotropic given by (2.10) if and only if the H-curvature satisfies the following:

$$
H_{i j}=\frac{n+1}{6} \theta F_{y^{i} y^{j}},
$$

where $\theta=\theta_{i}(x) y^{i}$ is a 1-form on $M$.

See Section 2 for the definition of the $H$-curvature. Say a Finsler metric $F$ has almost vanishing $H$-curvature if its $H$-curvature is given by $(1.1)[\mathbf{1 1}, \mathbf{1 3}]$. By using Theorem 1.1 and Proposition 3.2, we obtain a new and simple proof of Theorem 1.2 (see Section 5).

Riemannian metrics are a special case of Finsler metrics, namely Finsler metrics with the quadratic restriction. Call a geometric quantity on a Finsler manifold non-Riemannian if it vanishes for a Riemannian metric. For instance, the (mean) Cartan torsion, the $S$-curvature and the $H$-curvature are all non-Riemannian [6,7]. In Section 6, we show the following:

THEOREM 1.3. For a Finsler manifold with dimension $n=3$, the $\hat{C}$-curvature is a non-Riemannian quantity.

2. Preliminaries. Let $(M, F)$ be a Finsler manifold of dimension $n \geq 3$. In a standard local coordinate system $\left(x^{i}, y^{i}\right)$ in $T M, F=F(x, y)$ is a function of $\left(x^{i}, y^{i}\right)$. Let

$$
g_{i j}(x, y):=\frac{1}{2}\left[F^{2}\right]_{y^{i} y^{j}}(x, y)
$$


and $\left(g^{i j}\right):=\left(g_{i j}\right)^{-1}$. Let $R_{j}^{i}$ denote the Riemannian curvature of $F$ [7]. The $\hat{C}$-curvature is defined by $\hat{C}=\hat{C}_{i j} d x^{i} \otimes d x^{j}$ where

$$
\hat{C}_{i j}=R_{i j}+\frac{1}{n-2}\left(y_{i} R i c_{j}+y_{j} R i c_{i}-g_{i j} R i c-R i c_{i j} F^{2}\right)+\frac{S F^{2}}{(n-1)(n-2)} h_{i j},
$$

where $R_{i j}:=g_{i k} R_{j}^{k}$ is the flag curvature tensor of $F, h_{i j}:=F F_{y^{i} y^{j}}$ is the angular metric of $F[\mathbf{1 0}]$ and

$$
\begin{array}{r}
R i c:=R_{j}^{j}, \\
\operatorname{Ric}_{i}:=\frac{1}{2} \operatorname{Ric}_{y^{i}}, \\
\operatorname{Ric}_{i j}=\frac{1}{2} \operatorname{Ric}_{y^{i} y^{j}}, \\
y_{i}:=g_{i j} y^{j}, \\
S:=g^{i j} \operatorname{Ric}_{i j} .
\end{array}
$$

$F$ is of scalar flag curvature with flag curvature $K$ is equivalent to the following equation (see [6, page 110]):

$$
R_{j}^{i}=K F^{2} h_{j}^{i}
$$

where

$$
h_{j}^{i}=\delta_{j}^{i}-F^{-2} g_{j k} y^{k} y^{i}=g^{i k} h_{k j} .
$$

It is easy to see that (2.7) holds if and only if

$$
R_{i j}=K F^{2} h_{i j}
$$

In Sections 4 and 5, we will consider Finsler metrics of weakly isotropic flag curvature defined as follows:

$$
K=\frac{\theta}{F}+\sigma
$$

where $\sigma=\sigma(x)$ is a scalar function and $\theta=\lambda_{i}(x) y^{i}$ is a 1 -form.

The $H$-curvature $\mathbf{H}_{y}=H_{i j} d x^{i} \otimes d x^{j}$ is defined by

$$
H_{i j}=E_{i j \mid k} y^{k}
$$

where "I" denotes the covariant horizontal derivatives and $E_{i j}$ denote the mean Berwald curvature of $F[\mathbf{8}, \mathbf{1 3}]$. The $H$-curvature vanishes for a $R$-quadratic Finsler metric $[7,9]$.

3. Finsler metrics of scalar flag curvature. Assume that $F$ is of scalar curvature, that is, the flag curvature $K=K(x, y)$ is a scalar function on $T M \backslash\{0\}$. Using (2.2) and (2.7), we obtain the Ricci scalar Ric is given by

$$
\text { Ric }=(n-1) K F^{2},
$$


where we have used $F^{-2} g_{\ddot{y}} y^{i} y^{j}=1$. We use the following notations:

$$
K_{i}=K_{y^{i}}, \quad K_{\ddot{j}}=K_{y^{i} y^{j}} .
$$

By (2.3) and (3.1) we get

$$
R i c_{j}=\frac{n-1}{2}\left[K F^{2}\right]_{y^{j}}=\frac{n-1}{2}\left(K_{j} F^{2}+2 K y_{j}\right),
$$

where we have used the fact

$$
y_{j}=F F_{y^{j}}=\left(\frac{F^{2}}{2}\right)_{y^{j}} .
$$

Together with (2.4) we obtain

$$
R i c_{i j}=\frac{n-1}{2}\left(K_{i j} F^{2}+2 K_{i} y_{j}+2 K_{j} y_{i}+2 K g_{i j}\right),
$$

where we have used $\left(y_{j}\right)_{y^{i}}=\left(\frac{F^{2}}{2}\right)_{y^{j} y^{i}}=g_{i j}$. Note that the flag curvature $K$ is homogeneous of degree zero with respect to $y$. It follows that

$$
K_{j} y^{j}=0 .
$$

Using (2.6), (3.4) and (3.5), we obtain the scalar curvature $S$ is determined by

$$
S=\frac{n-1}{2}\left(g^{i \ddot{j}} K_{i j} F^{2}+4 K_{j} y^{j}+2 n K\right)=n(n-1) K+\frac{(n-1)^{2}}{2} F^{2} \Psi,
$$

where $\Psi:=\frac{1}{n-1} g^{i j} K_{i j}$. Together with (2.1), (2.9), (3.2) and (3.4), we get

$$
\begin{aligned}
\hat{C}_{\ddot{j}}= & K F^{2} h_{i j}+\frac{n-1}{2(n-2)}\left(4 K y_{i} y_{j}-K_{i} y_{j} F^{2}-K_{j} y_{i} F^{2}-4 K F^{2} g_{i j}-K_{i j} F^{4}\right) \\
& +\frac{n-1}{2(n-2)} \Psi F^{4} h_{i j}+\frac{n K}{n-2} F^{2} h_{i j} .
\end{aligned}
$$

Note that $y_{i} y_{j}-F^{2} g_{i j}=-F^{2} h_{i j}$. It follows that

$$
\begin{aligned}
\hat{C}_{i j}= & K F^{2} h_{i j}-\frac{n-1}{2(n-2)} F^{2}\left(4 K h_{i j}+K_{i} y_{j}+K_{j} y_{i}+K_{i j} F^{2}\right) \\
& +\frac{n-1}{2(n-2)} \Psi F^{4} h_{i j}+\frac{n K}{n-2} F^{2} h_{i j} \\
= & \frac{n-1}{2(n-2)}\left(\Psi F^{2} h_{i j}-K_{i} y_{j}-K_{j} y_{i}-K_{i j} F^{2}\right) F^{2} .
\end{aligned}
$$

Hence, we have the following:

Lemma 3.1. Let $(M, F)$ be an $n(\geq 3)$-dimensional Finsler manifold of scalar curvature with flag curvature $K(x, y)$. Then $F$ has vanishing $\hat{C}$-curvature if and only if in any standard local coordinate system

$$
\Psi F^{2} h_{i j}=K_{i} y_{j}+K_{j} y_{i}+K_{i j} F^{2},
$$

where $\Psi:=\frac{1}{n-1} g^{i j} K_{i j}$. In particular, $F$ has vanishing $\hat{C}$-curvature if $F$ has isotropic (or constant) flag curvature. 
A Finsler metric $F$ is said to be of isotropic curvature if the flag curvature $K(P, y)=$ $K(x)$ is a scalar function on $M$. In particular, $F$ is said to have constant (flag) curvature if the flag curvature $\mathbf{K}(P, y)=$ constant. Now we are going to establish an important equation between the flag curvature, the $\hat{C}$-curvature and the $H$-curvature.

Proposition 3.2. Let $F$ be a Finsler metric of scalar flag curvature on an n-manifold $M$. Then the flag curvature, $\hat{C}$-curvature and the H-curvature satisfy

$$
\hat{C}_{i j}=\frac{n-1}{2(n-2)}\left(\Psi F^{3} F_{y^{i} y^{j}}+\frac{6 H_{i j}}{n+1}\right) F^{2},
$$

where $\Psi:=\frac{1}{n-1} g^{\ddot{j}} K_{\ddot{j}}$.

Proof. A direct calculation yields (cf [7, (3.26)])

$$
0=6 H_{i j}+(n+1) F\left[(F K)_{y^{i} y^{j}}-K F_{y^{i} y^{j}}\right]=6 H_{i j}+(n+1)\left(K_{i} y_{j}+K_{j} y_{i}+K_{i j} F^{2}\right) .
$$

It follows that

$$
-\left(K_{i} y_{j}+K_{j} y_{i}+K_{i j} F^{2}\right)=\frac{6 H_{i j}}{n+1} .
$$

Plugging this into (3.6) yields (3.8).

4. Proof of theorem 1.1. First suppose that $F$ has weakly isotropic flag curvature, i.e. (2.10) holds. Differentiating (2.10) with respect to $y^{i}$, we obtain

$$
K_{i}=\frac{\lambda_{i}}{F}+\frac{\theta l_{i}}{F^{2}}
$$

where $l_{i}:=F_{y^{i}}=F^{-1} y_{i}$. Moreover

$$
K_{i j}=-\frac{\lambda_{i} l_{j}}{F^{2}}-\frac{\lambda_{j} l_{i}+\theta F_{y^{i} y^{j}}}{F^{2}}+\frac{2 \theta l_{i} l_{j}}{F^{3}}=\frac{\theta\left(3 l_{i} l_{j}-g_{i j}\right)-\left(\lambda_{i} l_{j}+\lambda_{j} l_{i}\right)}{F^{3}},
$$

where we have used the fact

$$
g_{i j}=h_{i j}+l_{i} l_{j}
$$

It follows that

$$
\Psi=-\frac{\theta}{F^{3}} .
$$

From (4.1), (4.2) and (4.3) we get

$$
K_{i} y_{j}+K_{j} y_{i}+K_{i j} F^{2}=\frac{\theta\left(3 l_{i} l_{j}-g_{i j}\right)-2 \theta l_{i} l_{j}}{F}=-\frac{\theta}{F} h_{i j} .
$$

It follows from (4.4) and (4.5) that

$$
\Psi F^{2} h_{i j}=-\frac{\theta}{F} h_{i j}=K_{i} y_{j}+K_{j} y_{i}+K_{i j} F^{2} .
$$

By Lemma 3.1, $F$ has vanishing $\hat{C}$-curvature. 
Conversely, suppose that $\hat{C}=0$. Then (3.7) holds. Differentiating (3.7) with respect to $y^{k}$, we obtain

$$
\Psi_{y^{k}} F^{2} h_{i j}+2 \Psi h_{i j} y_{k}+\Psi F^{2}\left(h_{i j}\right)_{y^{k}}=g_{i k} K_{j}+y_{i} K_{j k}+g_{j k} K_{i}+y_{j} K_{i k}+2 y_{k} K_{i j}+F^{2} K_{i j k},
$$

where

$$
K_{\ddot{j} k}:=\left(K_{i j}\right)_{y^{k}}=K_{y^{i} y^{j} y^{k}}
$$

is totally symmetric. Direct computations yield

$$
\begin{gathered}
g^{\ddot{j}} h_{i j}=n-1, \\
K_{\ddot{i} j} y^{i}=-K_{j}, \\
\left(h_{i j}\right)_{y^{k}}=2 C_{\ddot{j} k}-F^{-2} y_{j} h_{i k}-F^{-2} y_{i} h_{j k},
\end{gathered}
$$

where $C_{i j k}$ is the Cartan torsion [6]. Contracting (4.10) with $g^{i j}$ yields

$$
g^{i j}\left(h_{i j}\right)_{y^{k}}=2 I_{k}
$$

where $I_{k}$ is the mean Cartan torsion [6] and we have used the fact

$$
h_{i j} y^{i}=0 .
$$

Contracting (4.6) with $g^{i j}$ gives, by (4.8), (4.9) and (4.11),

$$
(n-1) \Psi_{y^{k}}+2 \Psi I_{k}=g^{i j} K_{\ddot{j} k} .
$$

Since $\Psi$ is homogeneous of degree -2 with respect to $y$, we have $\Psi_{y^{k}} y^{k}=-2 \Psi$. Together with (2.8) yields

$$
\Psi_{y^{k}} h_{j}^{k}=\Psi_{y^{j}}+2 F^{-2} y_{j} \Psi
$$

Contracting (4.10) with $g^{i k}$ yields

$$
g^{i k}\left(h_{i j}\right)_{y^{k}}=2 I_{j}-(n-1) F^{-2} y_{j},
$$

where we have made use of (4.8) and (4.12). Contracting (4.6) with $g^{i k}$ gives, by (3.5), (4.9) and (4.12),

$$
\Psi_{y^{k}} F^{2} h^{k}+\Psi F^{2} g^{i k}\left(h_{i j}\right)_{y^{k}}=n K_{j}+(n-1) y_{i} \Psi-2 K_{j}+F^{2} g^{i k} K_{i j k} .
$$

Plugging (4.14) and (4.15) into (4.16) yields

$$
F^{2}\left(\Psi_{y^{j}}+2 F^{-2} y_{j} \Psi\right)+\Psi F^{2}\left[2 I_{j}-(n-1) F^{-2} y_{j}\right]=(n-2) K_{j}+(n-1) y_{i} \Psi+F^{2} g^{i k} K_{i j k} .
$$

Taking this together with (4.13) yields

$$
(n-2)\left(F^{2} \Psi_{y^{j}}+K_{j}+2 y_{j} \Psi\right)=0 .
$$


It follows from (4.17) that

$$
\left(F^{2} \Psi+K\right)_{y^{j}}=F^{2} \Psi_{y^{j}}+2 y_{j} \Psi+K_{y^{j}}=0 .
$$

Thus

$$
\sigma:=F^{2} \Psi+K
$$

is a scalar function on $M$. Plugging (4.18) into (3.7) yields

$$
[\sigma(x)-K] h_{i j}=K_{i} y_{j}+K_{j} y_{i}+K_{i j} F^{2} .
$$

By using (3.3), (4.19) and the definition of the angular metric we get

$$
F_{y^{i}} K_{y^{j}}+F_{y^{j}} K_{y^{i}}+F K_{y^{i} y^{j}}+(K-\sigma) F_{y^{i} y^{j}}=0 .
$$

This implies that

$$
\begin{aligned}
{[(K-\sigma) F]_{y^{i} y^{j}} } & =\left[K_{y^{i}} F+(K-\sigma) F_{y^{i}}\right]_{y^{j}} \\
& =K_{y^{i} y^{j}} F+K_{y^{i}} F_{y^{j}}+K_{y^{j}} F_{y^{i}}+(K-\sigma) F_{y^{i} y^{j}}=0 .
\end{aligned}
$$

Note that $(K-\sigma) F$ is homogeneous of degree one with respect to $y$. Together with (4.20) we obtain $(K-\sigma) F$ is a 1 -form

$$
(K-\sigma) F=\lambda_{i}(x) y^{i}=\theta .
$$

We get that $K=\theta / F+\sigma$.

5. An alternative proof of the Najafi-Shen-Tayebi' theorem. In this section, we are going to give a new proof of Theorem 1.2 (see Section 1) using Theorem 1.1 and the important identity (3.8).

5.1. Proof of Theorem 1.2. First suppose that $H$ is almost vanishing given by (1.1). Plugging (1.1) into (3.9) yields

$$
\theta F_{y^{i} y^{j}}+K_{i} y_{j}+K_{j} y_{i}+K_{i j} F^{2}=0 .
$$

Contracting (5.1) with $g^{i j}$ gives, by (3.5) and (4.8), $(n-1)\left(\frac{\theta}{F}+F^{2} \Psi\right)=0$. It follows that

$$
\theta=-F^{3} \Psi
$$

Plugging this into (1.1) yields $H_{i j}=-\frac{n+1}{6} \Psi F^{3} F_{y^{i} y^{j}}$. Substituting this into (3.8) gives $\hat{C}=0$. By Theorem 1.1,

$$
K=\frac{\tilde{\theta}}{F}+\sigma,
$$

where $\sigma=\sigma(x)$ is a scalar function and $\tilde{\theta}=a_{i}(x) y^{i}$ is a 1 -form on $M$. By (4.4) we arrive at the following identity

$$
\Psi=-\frac{\tilde{\theta}}{F^{3}} .
$$


Plugging this into (5.2) yields $\tilde{\theta}=\theta$. We conclude that the flag curvature is weakly isotropic given by (2.10).

Conversely, suppose that $K=\frac{\theta}{F}+\sigma$ where $\sigma=\sigma(x)$ is a scalar function and $\theta=\lambda_{i}(x) y^{i}$ is a 1 -form on $M$. From (4.4) we deduce that

$$
\Psi=-\frac{\theta}{F^{3}} .
$$

By Theorem 1.1, $F$ has vanishing $\hat{C}$-curvature. Together with (3.8) we obtain

$$
H_{i j}=-\frac{n+1}{6} \Psi F^{3} F_{y^{i} y^{j}} .
$$

Plugging (5.3) into (5.4) yields (1.1).

6. Three-dimensional Finsler manifold. In Finsler geometry, there are several important non-Riemannian quantities: the mean Cartan torsion $\mathbf{I}$, the Cartan torsion $\mathbf{C}$ and the $H$-curvature $\mathbf{H}$, etc [6,7]. They all vanish for Riemannian metrics, hence they said to be non-Riemannian. In this section, we are going to show the following:

THEOREM 6.1. For a Finsler manifold with dimension $n=3$, the $\hat{C}$-curvature is a non-Riemannian quantity.

Proof. Assume that $(M, F)$ is a Riemannian manifold. Then then the flag curvature tensor $R_{i j}$ is given by

$$
R_{i j}=R_{k \ddot{j} l}(x) y^{k} y^{l}
$$

where $R_{k i j l}(x)$ is the Riemannian curvature of $F$. It follows that, from (2.2) and (2.4),

$$
R i c=R_{i j}(x) y^{i} y^{j}
$$

and

$$
\operatorname{Ric}_{i j}=R_{\ddot{j}}(x),
$$

where $R_{i j}(x)$ is the Ricci tensor of $F$. By using (2.6) and (6.1) we have

$$
S=R
$$

where $R$ is the scalar curvature of Riemannian metric $F$. Plugging (6.1)-(6.4) into (2.1) yields

$$
\begin{aligned}
\hat{C}_{i j}= & R_{k i j l}(x) y^{k} y^{l}+\frac{R}{(n-1)(n-2)}\left(g_{i j} g_{k l} y^{k} y^{l}-g_{i l} g_{k j} y^{k} y^{l}\right) \\
& -\frac{1}{n-2}\left(R_{i j} F^{2}-R_{i l} g_{k j} y^{k} y^{l}-R_{j l} g_{k i} y^{k} y^{l}+R_{k l} g_{i j} y^{k} y^{l}\right)=C_{k i j l} y^{k} y^{l},
\end{aligned}
$$

where $C_{k i j l}$ is the Weyl conformal curvature tensor [2]. Now our conclusion is an immediate consequence of $C_{k i j l} \equiv 0$ for a 3-dimensional Riemannian manifold [2, Proposition 3.3.9]. 


\section{REFERENCES}

1. H. Akbar-Zadeh, Sur les espaces de Finsler a courbures sectionnelles constants, Bull. Acad. Roy. Bel. Bull. Cl. Sci. 74(5) (1988), 281-322.

2. Z. Bai, Y. Shen, N. Shui and X. Guo, An introduction to Riemann geometry (Higher Education Press, 2004).

3. B. Chen and L. Zhao, A note on Randers metrics of scalar flag curvature, Cana. Math. Bull. to appear.

4. X. Chen, X. Mo and Z. Shen, On the flag curvature of Finsler metrics of scalar curvature, J. London Math. Soc. 68(2) (2003), 762-780.

5. X. Cheng and Z. Shen, Randers metrics of scalar flag curvature, J. Aust. Math. Soc. 87 (2009), 359-370.

6. S. S. Chern and Z. Shen, Riemann-Finsler geometry, in Nankai tracts in mathematics, 6 (World Scientific Publishing Co. Pte. Ltd., Hackensack, NJ, 2005), x+192.

7. X. Mo, On the non-Riemannian quantity $H$ for a Finsler metric, Diff. Geom. Appl. 27 (2009), 7-14.

8. B. Najafi, Z. Shen and A. Tayebi, Finsler metrics of scalar flag curvature with special non-Riemannian curvature properties, Geom. Dedicata 131 (2008), 87-97.

9. Z. Shen, R-quadratic Finsler metrics, Publ. Math. Debrecen. 58 (2001), 263-274.

10. Z. Shen, Differential geometry of spray and Finsler spaces (Kluwer Academic Publishers, 2001), 258.

11. Z. Shen, On some non-Riemannian quantities in Finsler geometry, Cana. Math. Bull. (2011), to appear.

12. Z. Shen and H. Xing, On Randers metrics with isotropic S-curvature, Acta Math. Sin. (Engl. Ser.) 24(2008), 789-796.

13. D. Tang, On the non-Riemannian quantity $H$ in Finsler geometry, Diff. Geom. Appl. 29 (2011), 207-213. 